\title{
CICLO BIOLÓGICO Y EXPLORACIÓN DE PARASITISMO EN SPECULARIUS IMPRESSITHORAX (PIC) 1932 (COLEOPTERA: BRUCHIDAE) EN MÉXICO
}

\section{Angélica V. RÍOS R. ${ }^{1}$, Jesús ROMERO N. ${ }^{1}$, José Luis CARRILlO S. ${ }^{1}$, Hiram BRAVO M. ${ }^{1}$, Jorge VERA G. ${ }^{1}$ \& SAMUEL RAMÍREZ A. ${ }^{2}$}

\author{
${ }^{1}$ Instituto de Fitosanidad, Colegio de Postgraduados, km 36.5 carr. fed. México-Texcoco \\ Montecillo, Estado de México, C.P. 56230, México; <riosreyes@colpos.mx>, <jnapoles@colpos.mx>, \\ <josecarr@colpos.mx>,<bravomj@colpos.mx> \\ ${ }^{2}$ Departamento de Parasitología Vegetal, Universidad Autónoma Chapingo, Estado de México. \\ <samuelram@prodigy.net.mx>
}

Recibido: 26/09/2013; aceptado:28/11/2014

Ríos R., A. V., Romero N., J., Carrillo S., J. L., Bravo M., H., Vera G., J., \& Ramírez A., S. 2015. Ciclo biológico y exploración de parasitismo en Specularius impressithorax (Pic) 1932 (Coleoptera: Bruchidae) en México. Acta Zoológica Mexicana (n. s.), 31(1): 27-35.

RESUMEN. El brúquido Specularius impressithorax es un insecto de origen africano cuyo desplazamiento se ha dado de manera importante a nivel mundial debido a que se le ha encontrado en países como la India, Estados Unidos (Hawaii, California) y actualmente México, en este último desplazándose a varios estados de la República, en gran parte debido a que su principal fuente de alimento son las semillas de plantas del género Erythrina, las cuales se encuentran abundantemente en zonas con climas desde semiáridos hasta secos o templados; el clima en México y la diversidad de especies de Erythrina ha favorecido al establecimiento del insecto y por lo tanto el ataque a nuevos hospederos. Para el estudio del ciclo biológico del insecto se realizó la cría del mismo y se cuantificó el número de huevos depositados y el tiempo requerido para cada etapa de desarrollo, todo ello a temperatura ambiente $\left(18-22^{\circ} \mathrm{C}\right)$ y temperatura controlada de $25^{\circ} \mathrm{C} \pm 2{ }^{\circ} \mathrm{C}$; también se observó la polifagia hacia nuevos hospederos y la exploración de parasitismo en $S$. impressithorax. Los resultados mostraron que $S$. impressithorax en condiciones controladas de laboratorio presenta una disminución en la oviposición de huevos viables, en comparación con la temperatura ambiente, en donde la mayoría de los huevos llegaron a ser fértiles y la población llegó a estado adulto. Insectos vírgenes de $S$. impressithorax fueron colocados en contenedores donde se les agregó alimento de diferentes especies de Erythrina originarias de México y se evaluó la preferencia alimenticia. Se observó que fue capaz de invadir nuevos hospederos y de ellos emergieron adultos. Al realizar las colectas en donde se presentaban semillas de eritrinas infestadas por S. impressithorax se observó la presencia de parasitoides de huevos, éstos se identificaron como Uscana sp. (Hymenoptera: Trichogrammatidae); sin embargo, no se han podido obtener formas adultas libres para la corroboración de la especie; el segundo enemigo natural encontrado correspondió a Entedeon erythrinae Gumovsky y Ramadan (Hymenoptera: Eulophidae), se trata de un parasitoide de huevo-larva del brúquido. Los datos anteriores indican que la infestación hacia nuevos hospederos de eritrinas y el incremento de su dispersión será inminente y que hace falta realizar estrategias de control del brúquido; el control biológico se puede incluir en su manejo y así evitar el deterioro del germoplasma de los hospederos, mismos que realizan funciones importantes en la ecología, la etnobótanica y la ciencia.

Palabras clave: Gorgojo de las semillas, enemigos naturales, plaga exótica.
Ríos R., A. V., Romero N., J., Carrillo S., J. L., Bravo M., H., Vera G., J., \& Ramírez A., S. 2015. Life cycle and exploration of parasitism in Specularius impressithorax (Pic) 1932 (Coleoptera: Bruchidae) from Mexico. Acta Zoológica Mexicana (n. s.), 31(1): 27-35.

ABSTRACT. Specularius impressithorax is a bruchid of African origin whose displacement has been worldwide, it has been found in India, United States (Hawaii, California), and currently in Mexico. In the latter country, it has moved to several states because of its food source, that is the Erythrina's seeds, which are found abundantly in climatic zones that goes from semiarid to dry or temperate; the climate and the Erythrina species diversity found in Mexico are favorable for its establishment and the attack to new hosts. For the study of the insect life cycle, breeding was done, the number of eggs laid was recorded, as well as the time required for each developmental stage; also the polyphagy was measured by offering to it new hosts, and an exploration of parasitism in $S$. impressithorax, all of this at environmental temperature $\left(18-22^{\circ} \mathrm{C}\right)$ and controlled temperature of $25^{\circ} \mathrm{C} \pm$ $2{ }^{\circ} \mathrm{C}$. The results showed that $\mathrm{S}$. impressithorax in the laboratory with controlled conditions undergo a decrease in the oviposition of fertile eggs in comparison to the environmental temperature where most of the eggs become fertile and reached the adult stage. Virgin insects of $S$. impressithorax were placed in containers where they were fed with different species of Erythrina native to Mexico and the food preferences was evaluated. It was observed that they were capable to invade new hosts, from which adults emerged. The collects were carried out where seeds infested by $S$. impressithorax were present, eggs of the bruchid attacked by a parasite was observed, this was identified as Uscana sp. (Hymenoptera: Trichogrammatidae); however, free adult forms could not be obtained for corroboration of the species. A second natural enemy was determined as Entedeon erythrinae Gumovsky \& Ramadan (Hymenoptera: Eulophidae) that is an egg-larvae parasitoid of bruchids. Previous data show that infestation to new Erythrina hosts and the increase of its dispersion will be imminent, and that it is necessary to implement control strategies for the bruchid; biological control can be included in its management and thus prevent the deterioration of the host germplasm, which play important functions in the ecology, the ethnobotany, and science.

Key words: Seed beetle, natural enemies, exotic pests. 


\section{INTRODUCCIÓN}

Actualmente, un aspecto importante en México es la protección y manejo apropiado de las reservas naturales para preservar, sobre todo, el reservorio del germoplasma, mismo que asegurará la subsistencia de las especies en el futuro; además, se requiere promover la ecología y el aprovechamiento sustentable de las especies, además, esto conlleva a conocer el papel que realizan las especies en el ecosistema y las aplicaciones que pueda obtener el hombre de ellas. De las especies de plantas que existen, las leguminosas han desempeñado un papel importante en la rehabilitación de sitios dañados por erosión o por falta de un manejo adecuado; su uso también se extiende a varias aplicaciones más como la ecológica, étnica, médica, forestal, ganadera, ornamental, entre otras.

En el caso específico de Erythrina breviflora DC. y E. americana Mill., se ha registrado que las semillas presentan elevadas cantidades de alcaloides; sin embargo, Sotelo et al. (1993) indican que después de un proceso de detoxificación se pueden utilizar como alimento y de esta manera aprovechar su alto contenido de proteína. Desde el punto de vista médico, existen registros de que estas plantas se pueden utilizar como relajantes del sistema nervioso periférico y central, así como coadyuvantes en anestesia (García-Mateos et al. 2000). En México y países de América Central se usan como forraje y alimento (Sotelo et al. 1993; Kass, 1994), mientras que en Costa Rica, los usos que se le dan a varias especies de Erythrina son diversos, pudiendo emplearse como plantas fijadoras de nitrógeno y como plantas madre para cafetales; para tal efecto se ha usado la especie E. poeppigiana (Walp.) O. F. Cook, y también como forraje para bovinos, ayudando a incrementar la masa corporal de los animales por el alto contenido de proteína de la planta (Informe 217s 1989). Las especies de Erythrina se distribuyen en todo el mundo, pero en México y Centroamérica se localiza la mayor concentración de ellas (Neill 1993).

En 1932, Pic describe a una especie africana muy típica como Bruchus impressithorax; posteriormente, Bridwell (1938) describe a un insecto detectado en los Estados Unidos proveniente de Kenia en semillas de Erythrina como Specularius erythrinae, haciendo referencia al espejo o área lustrosa que presentaba en el pigidio el insecto y al nombre del hospedero; los ejemplares eran tan típicos que fue necesario hacer también la descripción de un nuevo género. Sin embargo, Bridwell ya había tenido nociones de estos especímenes con anterioridad: en 1924 los había observado infestando semillas de E. variegata L. en la India, mientras que en 1927 tuvo la oportunidad de revisar la colección del Museo Nacional de los Estados Unidos (USNM), en donde encontró insectos del mismo tipo obtenidos en semillas de E. abyssinica DC. de Tanganyika,
Tanzania, y en semillas de una Erythrina no determinada de Sumatra, Indonesia. Por su parte, Decelle (1951) notó que B. impressithorax y S. erythrinae correspondían a la misma especie, de tal manera que sinonimizó el segundo nombre y conservó al nuevo género, por lo cual el nombre válido para la especie en cuestión es Specularius impressithorax (Pic 1932).

En el año 2001 se registró la introducción de este insecto en Hawaii, EUA, atacando a una especie endémica, E. sandwicensis Degener; posteriormente se le observó alimentándose de E. variegata, una especie no nativa del sitio, la cual era cultivada en la isla, y para el año 2003 llegó a atacar a especies nativas y no nativas en dicha región (Medeiros et al. 2008). En 2004, Gulmahamad (2006), elaboró el primer reporte de $S$. impressithorax para el nuevo mundo, específicamente en California, EUA, en donde se le observó alimentarse de semillas de Erytrina spp.; hasta el momento se le ha registrado en eritrinas ornamentales en parques, áreas públicas y escuelas, y no se cuenta con información actualizada de que las especies nativas hayan sido invadidas. Romero et al. (2009) registraron la presencia de este brúquido para México, específicamente en Texcoco, Estado de México, y en el Distrito Federal en semillas de E. coralloides DC. y E. americana; para el año 2011 el insecto se había desplazado hacia los estados de Morelos y Veracruz, en donde se le registró alimentándose de semillas de E. americana (Ruiz et al. 2012).

Además de la amplia distribución del brúquido, no existen reportes sobre algún tipo de control biológico o manejo integrado para esta especie exótica, y sólo se menciona la presencia de tres especies de avispas (Bethylidae y Eulophidae) en la isla de Hawaii, EUA, que probablemente sean parasitoides de la larva del brúquido; también se indican registros de otro parasitoide identificado como Entedeon sp. (Hymenoptera: Eulophidae) en Sumatra, India (Medeiros et al. 2008). El trabajo más reciente sobre parasitoides se publicó en 2011 por Gumovsky y Ramadan, en donde se describe una nueva especie, Entedeon erythrinae Gumovsky y Ramadan, misma que fue registrada en Sudáfrica, Tanzania, Mozambique y Hawaii en EUA; se trata de un parasitoide que oviposita en los huevecillos de $S$. impressithorax y posteriormente se desarrolla en el interior de la larva, la cual nunca llega a pupar, de tal manera que los parasitoides emergen de los restos de la larva madura de S. impressithorax.

Debido a la amplia distribución que ha registrado este brúquido en diferentes partes del mundo y a los hábitos polífagos que presenta, los objetivos de este estudio fueron 1) conocer el ciclo biológico de S. impressithorax y el grado de polifagia que presenta, así como 2) detectar la presencia de sus posibles enemigos naturales y el status actual de su distribución en México. 


\section{MATERIALES Y MÉTODOS}

Ciclo de vida. Se recolectaron semillas de E. americana de árboles localizados en Texcoco, Estado de México. De los árboles se seleccionaron semillas infestadas para la obtención del pie de cría del insecto y semillas sanas para los experimentos; estas últimas se seleccionaron con base en la ausencia de huevos sobre las semillas, o bien, cuando las semillas estuvieran cubiertas por la vaina, siendo ambas características una evidencia de que aún no estaban infestadas. Para determinar el ciclo biológico del insecto se colocaron cinco semillas sanas de E. americana en cajas Petri de poliestireno cristal de 100 x15 mm, y se depositó una pareja de hembra y macho vírgenes para cuantificar la oviposición; se realizaron 16 repeticiones y se cuantificó diariamente el total de huevos fértiles e infértiles, es decir, la presencia o no de aserrín visible a través del corión, así como la longevidad de los adultos parentales. Posteriormente, se esperó la emergencia de los adultos de la primera generación, se observó y cuantificó la oviposición de los mismos y se esperó la emergencia de nuevos individuos hasta la última emergencia; se sexaron todas las generaciones nuevamente. La mitad de las cajas se mantuvo a temperatura ambiente oscilante $\left(18-22^{\circ} \mathrm{C}\right)$ con $8 \mathrm{~h}$ luz y $16 \mathrm{~h}$ oscuridad y la otra mitad fija $\left(25^{\circ} \mathrm{C}\right)$, con un fotoperiodo de $12 \mathrm{~h}$ luz-12 h oscuridad.

Se observó en laboratorio la capacidad de oviposición sobre semillas anteriormente utilizadas por S. impressithorax; el insecto deja una perforación al salir de la semilla, puede variar la cantidad de perforaciones dependiendo de la cantidad de insectos que logran completar su estado inmaduro hasta emerger; es por ello que se realizó el experimento de dejar que el insecto ovipositara varias veces y observar el desarrollo del insecto en cantidades de alimento limitado ya que en campo se ha observado una oviposición muy abundante sobre semillas de una temporada anterior que quedan sujetas al árbol. Para tal finalidad se colocaron cinco semillas en cajas Petri, esta cantidad se determinó debido a que la cantidad de semillas en una vaina de E. coralloides son aproximadamente cinco (se determinó esa cantidad debido a que en esta especie la vaina presenta un promedio de cinco semillas), para observar la sobreoviposición de las mismas y la emergencia de adultos.

Además, se observó de manera azarosa un poco del comportamiento del insecto al realizar la oviposición sobre la semilla.

Polifagia. Se realizaron bioensayos en los que se observó el grado de polifagia y preferencia de alimentación de $S$. impressithorax por diferentes especies de eritrinas, entre las cuales se encuentran dos especies ya registradas como hospederos (E. americana y E. coralloides), además de las siguientes que nunca se han registrado como hospederos: E. breviflora, E. chiapasana, E. fusca y E. leptorhiza, todas originarias de México. Se depositó una hembra y un macho (vírgenes) en un contenedor de plástico de dimensiones amplias para permitir la movilidad del insecto y con ventilación, junto con 3 semillas por especie de planta, y de cada una se hicieron 15 repeticiones, de estas, se agregaron 5 repeticiones en cada cámara de cría con temperatura controlada de $20^{\circ} \mathrm{C} \pm 2{ }^{\circ} \mathrm{C}, 25^{\circ} \mathrm{C} \pm 2{ }^{\circ} \mathrm{C}$ y $30^{\circ} \mathrm{C} \pm 2^{\circ} \mathrm{C}$ y $12 \mathrm{~h}$ de fotoperiodo; se cuantificó el número de huevos depositados por día, posteriormente se evaluó su fertilidad. El análisis estadístico de los datos se realizó con ANOVA comparando las temperaturas y el grado de oviposición y fertilidad de los huevos; la comparación de las medias para cada tratamiento se realizó con la prueba de Tukey (HSD), utilizando el programa SAS (2000).

Parasitismo. Para la exploración de parasitoides y su distribución se realizaron colectas del brúquido de 2010 a 2013 en diferentes entidades del país. Los datos específicos de colecta se mencionan a continuación: DISTRITO FEDERAL: Instituto de Biología, UNAM, 15/VI/2009, Aldrete A., Erythrina americana (100 ex. CEAM); Tlatelolco, Del. Cuauhtémoc, 15/IV/2007, Ojeda I., Erythrina sp. (100 ex. CEAM); Jardines de la Universidad Autónoma Metropolitana, 2/XII/2010, Erythrina coralloides; Escuela Primaria "Mariano Hidalgo", Colonia Santiago, Acahualtepec, Delegación Iztapalapa, 2252 m, Guillermo Romero Gómez, Erythrina americana, 19²1'23.2'” N, 9900'13.96', O (20 ex. CEAM). ESTADO DE MÉXICO: Colegio de Postgraduados, Montecillo, 16/II/2012, 2250 m, Ramírez S., A., Erythrina coralloides DC., 19²8'04.26', N, 98'53'52.18'” O (7 ex. CEAM); Colegio de Postgraduados, Montecillo, 4/XI/2011, $2250 \mathrm{~m}$, Ramírez S., A., Erythrina coralloides DC., 19²8'04.26”' N, 98 53'52.18', O (200 ex. CEAM); Colegio de Postgraduados, Montecillo, 6/I/2007, 2256 m, Rodríguez H. C., Erythrina coralloides DC., 19²7'45.73'” N, 98 54'13.03'’ O (1000 ex. CEAM); Montecillos, Estado de México, 2256 msnm, 19²7’45.73' ' N, 9854'13.03', O; Huexotla, Texcoco de Mora, 18/X/2012, Angélica Ríos, Erythrina americana L., 19²7’45.17', N, 9852'52.01'” O (100 ex. CEAM;). GUANAJUATO: A un costado de la carretera 45 Celaya-Villagrán, Zona Urbana, Celaya, Erythrina sp., Pedro de Jesús Parra Gil, 2/V/2012, 1757 msnm, Erythrina sp., 20³0'50.39' 'N, 10049'42.87'’O (30 ex. CEAM). HIDALGO: Jardín de un salón de fiestas, Pachuca de Soto, 20/VIII/12, Sergio Godínez Cortés, 2412 msnm, Erythrina coralloides DC, 2006'53.83'”N, 9843'20.93'’O (20 ex. CEAM). JALISCO: Centro Universitario de Ciencias Biológicas y Agronomía (CUCBA), Universidad de Guadalajara, hospedero Erythrina americana L, 14/XII/2011, 1650 m, Osiris Serrano Pine- 
da, 2074’ N, 10330’ O (51 ex. CEAM). MORELOS: Santa María Ahuacatitlán, km 67.6 carr. fed. MéxicoCuernavaca, 27/IV/2011, Romero N., J., Erythrina sp., (1 ex. CEAM). PUEBLA: Tehuacán; Jardines y alrededores del monumento al Ángel custodio del Parque Analco y del Centro recreativo y Cultural, Puebla, Erythrina coralloides, Sergio Godínez Cortés, 22/V/12, 2143 msnm, 1902'26,80' 'N, 98¹1'36.34'’O. NAYARIT: Unidad Académica de Agricultura, Universidad Autónoma de Nayarit, Tepic, 4/V/2013, Romero N., J., Erytrina sp. (1 semilla con huevecillos y opérculos de emergencia de $S$. impressithorax, CEAM). VERACRUZ: El Lencero, Emiliano Zapata, 25/III/2011, Hernández M., M. J., Erythrina americana MILL. (71 ex. CEAM).

De todas las localidades antes indicadas se colectaron semillas infestadas y se llevaron al laboratorio de Taxonomía del Colegio de Postgraduados. Las muestras fueron depositadas en cámaras de cría a una temperatura de $25 \pm 2^{\circ} \mathrm{C}$ con fotoperiodo de $12 \mathrm{~h}$. Periódicamente se inspeccionaban para observar alguna actividad parasítica; el resto del material se depositó en la Colección Entomológica del Instituto de Fitosanidad (CEAM) de la misma institución.

\section{RESULTADOS}

Ciclo biológico de Specularius impressithorax. A una temperatura controlada de $25^{\circ} \mathrm{C}$, se observó en promedio una oviposición de 8 a 10 huevos por pareja; sin embargo, se presentaron oviposiciones de hasta 22 huevos, de los cuales más del $90 \%$ fueron fértiles y de esos casi el 80\% llegó a etapa adulta; el máximo de huevos que una hembra puede depositar en una semilla es de 23. El estado inmaduro del brúquido desde su etapa de huevo hasta que emerge como adulto es de 40 a 60 días en promedio. La longevidad de las hembras fue de 22 días en promedio, aunque en algunas hembras su longevidad alcanzó hasta los 55 días, mientras que la longevidad de los machos resultó más corta o igual al promedio de las hembras; en lo que respecta a la relación hembra-macho, ésta fue de 2:1. A temperatura ambiente $\left(18-22{ }^{\circ} \mathrm{C}\right)$ la cantidad de huevos puede ser menor; sin embargo, la fertilidad de los mismos y la emergencia de adultos fueron mayores que a temperatura controlada, así como la longevidad de las hembras aumentó a temperatura controlada (Cuadro 1).

Las semillas de E. coralloides son dehiscentes (Neill 1993) es decir, que la vaina abre y expone a las semillas de manera parcial o total y sólo las semillas expuestas son susceptibles a que $S$. impressithorax oviposite; un aspecto importante de la relación insecto-planta es que algunas de las semillas caen pero otras permanecen adheridas a la vaina, misma que se encuentra sujeta en el árbol y puede permanecer ahí hasta el siguiente periodo de floración y fructificación. En estas circunstancias, dichas semillas pueden ser reutilizadas por los insectos para ovipositar nuevamente, de tal manera que existe un traslape de generaciones. Otra peculiaridad que exhibe esta especie de brúquido, es que puede responder a una restricción de alimento mediante la reducción de su tamaño corporal; en estas condiciones se observó una reducción del 45\% del tamaño del cuerpo. En relación al comportamiento de la oviposición de S. impressithorax, en condiciones controladas, se observó de manera azarosa, la oviposición de una hembra de $S$. impressithorax, la cual se realizó por la tarde, aproximadamente a las 18:00 hrs; la hembra explora la semilla y determina el sitio donde va a ser colocado el huevo. Antes de colocarlo, pasa sus mandíbulas sobre la testa por unos segundos, después gira y sobre el sitio donde realizó tal actividad deposita el huevo, mismo que se adhiere y endurece en poco tiempo.

Polifagia. En la comparación de las medias de Erythrina no mostraron diferencia significativa $(\mathrm{F}=3.61 \mathrm{gl}=12 \mathrm{y}$ $\mathrm{p}>0.0001$ ) en las temperaturas de $25^{\circ} \mathrm{C}$ y $30{ }^{\circ} \mathrm{C}$, debido a que los valores se encuentran dentro de la misma letra, sin embargo, en la temperatura de $20^{\circ} \mathrm{C}$ se presentó una diferencia en la especie de E. leptorhiza y E. breviflora, mostrando que la comparación de las medias fue diferente. Estadísticamente no hubo diferencia significativa, sin embargo, biológicamente la mayor cantidad de huevos ovipositados fue en la temperatura de $20^{\circ} \mathrm{C}$, mostrando una media de 0.92 en E. leptorhiza y una media menor en E. breviflora (Cuadro 2). El grado de polifagia observado

Cuadro 1. Desarrollo de Specularius impressithorax a temperatura ambiente y a temperatura controlada.

\begin{tabular}{lcc}
\hline Desarrollo & Temperatura ambiente & Temperatura controlada \\
& $\mathbf{1 8 - 2 2}{ }^{\circ} \mathbf{C} \pm \mathbf{2}{ }^{\circ} \mathbf{C}$ & 8 a 12 \\
\hline Oviposición & $<12$ huevos & $<90 \%$ \\
Fertilidad del huevo & $>90 \%$ & 40 a 60 días \\
Tiempo de emergencia de adultos & 30 a 40 días & 22 a 55 días \\
Longevidad de las hembras & 19 a 20 días & Igual o más corto que la hembra \\
Longevidad de los machos & Igual o más corto que la hembra & $2: 1$ \\
Relación hembra: macho & $2: 1$ & \\
\hline
\end{tabular}


Cuadro 2. Efecto de la temperatura sobre la cantidad de huevos ovipositados en diferentes especies de Erythrina.

\begin{tabular}{ccc}
\hline Temperatura & Media \pm EE* & Erythrina \\
\hline $20^{\circ} \mathrm{C}$ & $0.92 \pm 0.184 \mathrm{~A}$ & E. leptorhiza \\
$0.86 \pm 0.248 \mathrm{BA}$ & E. coralloides \\
$0.74 \pm 0.185 \mathrm{BAC}$ & E. americana \\
$0.60 \pm 0.364 \mathrm{BAC}$ & E. fusca \\
$0.28 \pm 0.027 \mathrm{BC}$ & E. chiapasana \\
& $0.20 \pm 0.035 \mathrm{C}$ & E. breviflora \\
& $0.48 \pm 0.271 \mathrm{~A}$ & E. coralloides \\
& $0.36 \pm 0.061 \mathrm{~A}$ & E. fusca \\
& $0.34 \pm 0.123 \mathrm{~A}$ & E. leptorhiza \\
& $0.28 \pm 0.109 \mathrm{~A}$ & E. chiapasana \\
& $0.22 \pm 0.061 \mathrm{~A}$ & E. americana \\
& $0.22 \pm 0.054 \mathrm{~A}$ & E. breviflora \\
& $0.30 \pm 0.087 \mathrm{~A}$ & E. breviflora \\
& $0.26 \pm 0.101 \mathrm{~A}$ & E. coralloides \\
& $0.24 \pm 0.015 \mathrm{~A}$ & E. americana \\
& $0.20 \pm 0.042 \mathrm{~A}$ & E. chiapasana \\
\hline
\end{tabular}

Valores dentro de la misma columna de cada temperatura seguidos por la misma letra no son significativamente diferentes, Tukey $(\alpha=0.05)$. Error Estandar*

sobre las diferentes especies de eritrina fue variable. Se observó que el brúquido ovipositó en todas las semillas de diferentes especies, aunque no todos los huevos eclosionaron y sólo algunas larvas penetraron a las semillas.

$\mathrm{Al}$ realizar el conteo de los huevos fértiles en relación con las temperaturas, se pudo observar que a una temperatura de $20^{\circ} \mathrm{C}$ la cantidad de huevos fértiles es del 56\%, mientras que a temperaturas de $25{ }^{\circ} \mathrm{C}$ y $30{ }^{\circ} \mathrm{C}$ fueron de $48 \%$ y $18 \%$, respectivamente. La mayor cantidad de larvas que llegaron al estadio 1 y penetraron a las semillas se observó a $25^{\circ} \mathrm{C}$, la menor fue a $30^{\circ} \mathrm{C}$. De las plantas hospederas en donde se ha obtenido en campo las colonias de brúquidos, las cuales son E. americana y E. coralloides, era de esperarse obtener la mayor cantidad de huevos fértiles, sin embargo los resultados fueron diferentes.

Parasitoides asociados a $S$. impressithorax (Cuadro 3). Se detectaron dos especies de parasitoides, uno de ellos se encontró en huevecillos de S. impressithorax; su reconocimiento se hizo al detectar que los huevecillos parasitados se tornaron obscuros, debido a que los miembros del género Uscana sp. (Hymenoptera: Trichogrammatidae) son precisamente de este color (Figuras 1a, 1b); sin embargo, no se pudieron obtener formas adultas para la corroboración de la especie. Las evaluaciones de parasitismo se pudieron registrar debido a la coloración de los huevos, ya que al momento del conteo se podían reconocer los huevos obscuros denotando parasitismo, o bien,
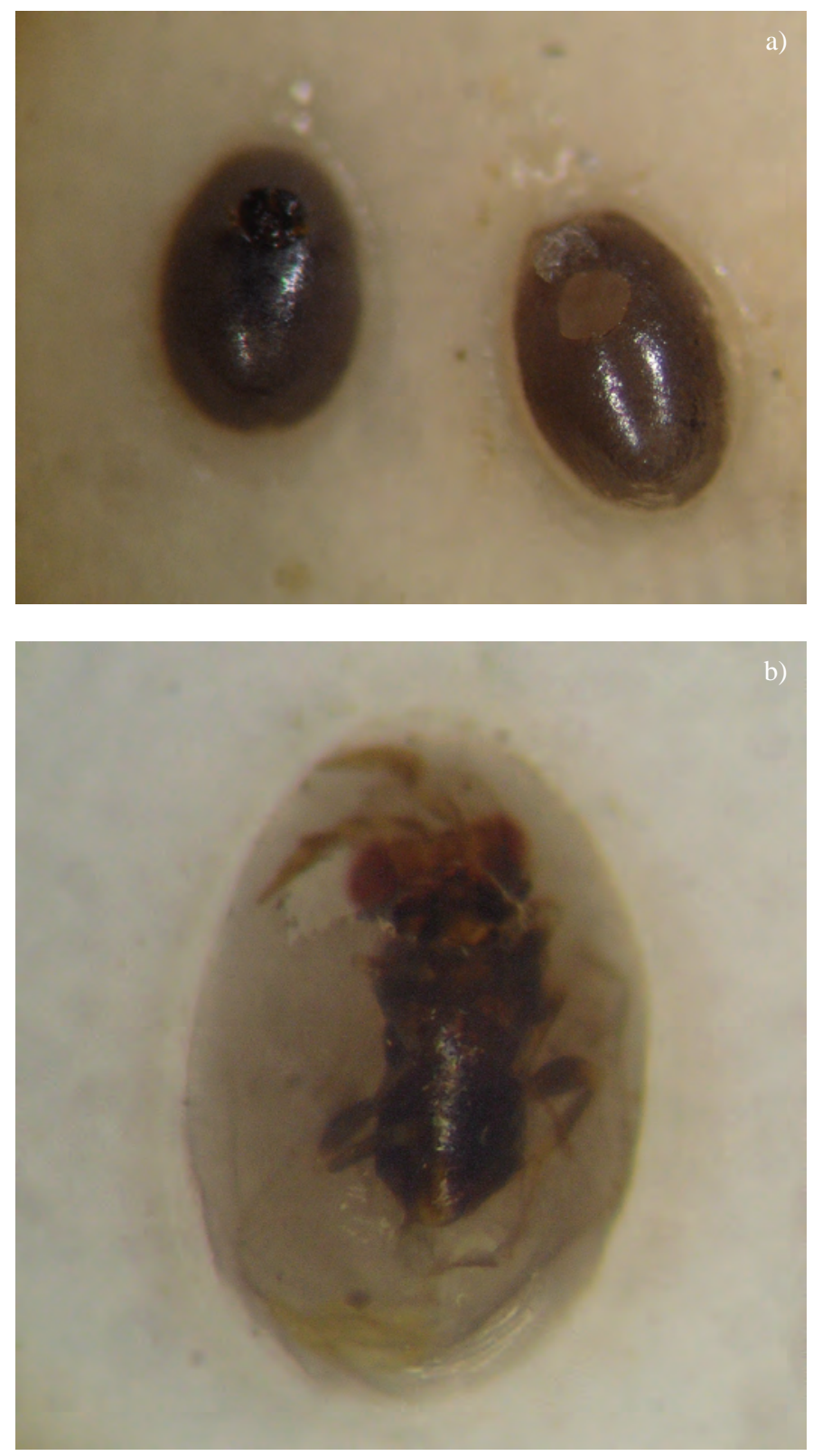

Figura 1. Huevecillos de Specularius impressithorax parasitados por Uscana sp. a) orificio de emergencia del parasitoide, b) parasitoide en el interior del huevecillo.

huevos con un orificio de emergencia del parasitoide, en ambos casos considerados como huevos parasitados. En la localidad de Huexotla, Texcoco de Mora, se registró un porcentaje de parasitismo de 3.87\%, en el Instituto de Biología, UNAM de 59.99\% y en el material proveniente de los jardines de la Universidad Autónoma Metropolitana de $32.02 \%$ (Cuadro 4).

En muestras de Huexotla, Texcoco de Mora, se encontraron adultos de un parasitoide determinado como Entedeon erythrinae Gumovsky y Ramadan (Hymenoptera: Eulophidae). El parasitoide se encontró en larvas de $S$. impressithorax mediante la inspección de algunas semi- 
Cuadro 3. Relación actual de hospederos registrados para Specularius Impressithorax en el mundo.

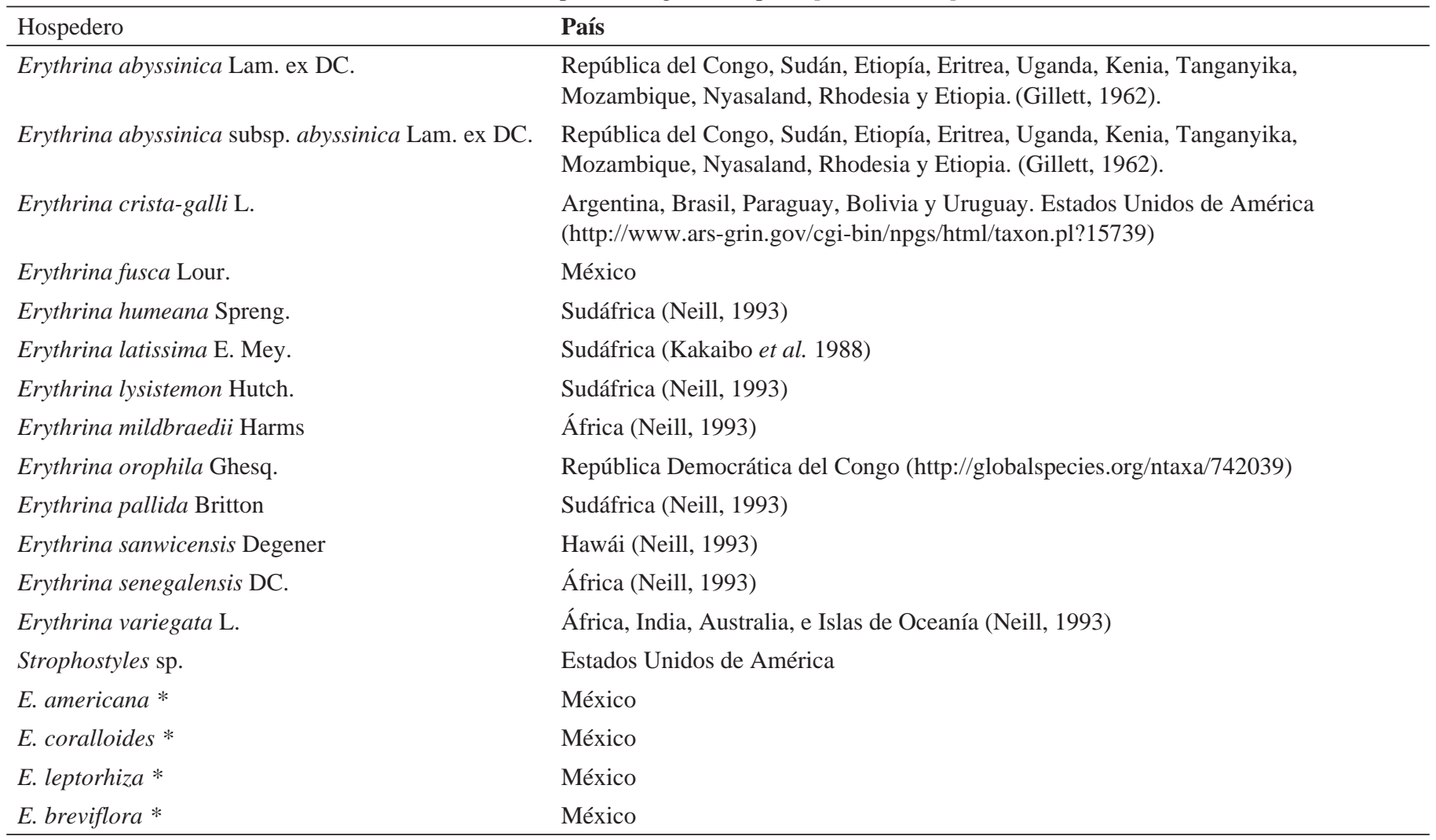

* Hospederos potenciales para Specularius impressithorax

Cuadro 4. Sitios de colecta donde se encontró presencia de parasitoides de huevo de Specularius impressithorax.* Hospederos potenciales para Specularius impressithorax.

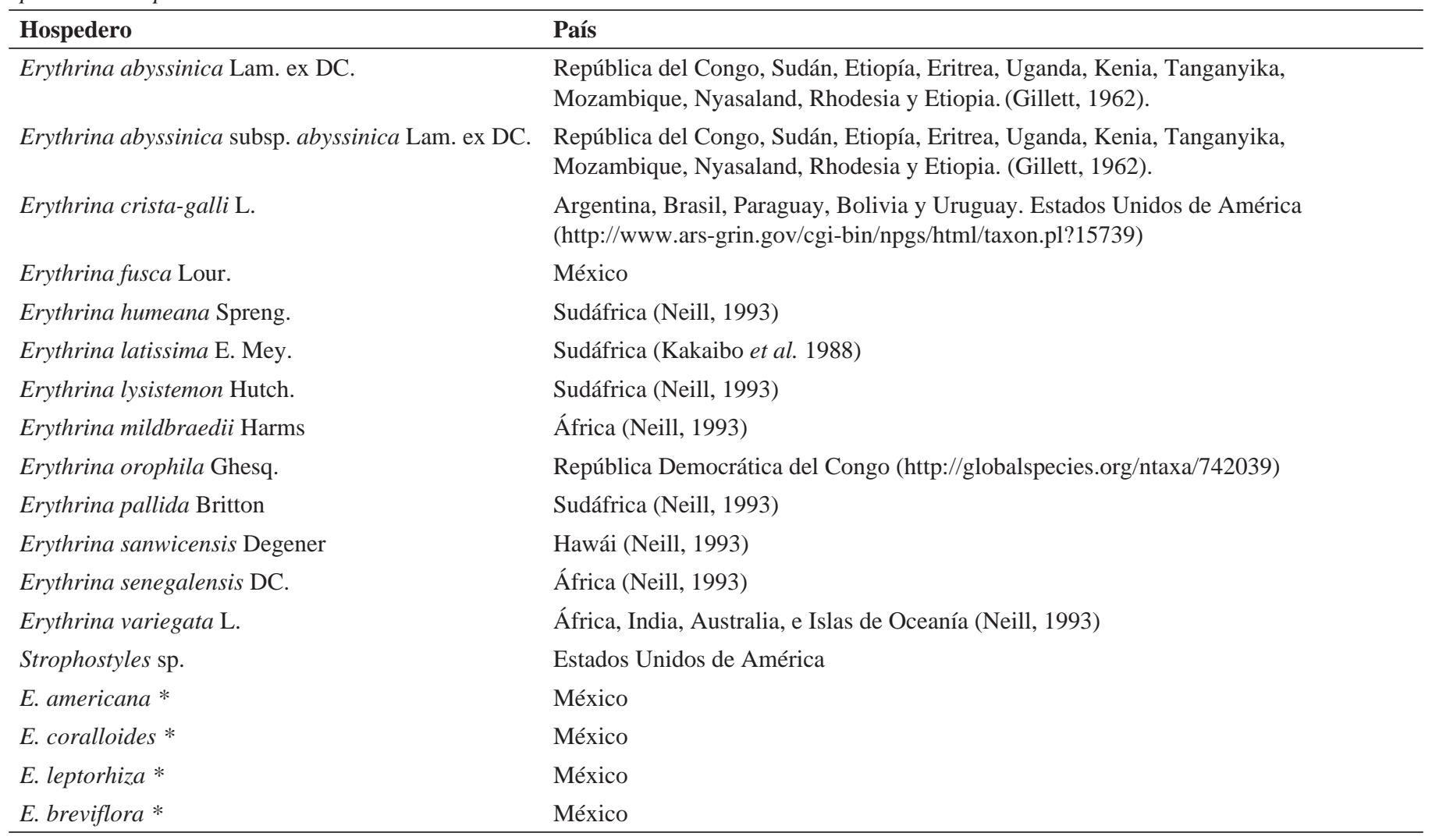


llas de E. coralloides (Figura 2), por lo que se cubrió el orificio de la semilla y se esperó a la emergencia de los parasitoides. Es posible que esta nueva interacción huésped-parasitoide se esté iniciando, ya que los parasitoides son los que finalmente deberían terminar el opérculo de emergencia en la semilla; sin embargo, debido a que la testa de la semilla es muy gruesa, ellos no pueden romperla y mueren en el interior de la semilla junto con el brúquido. Se localizaron dos semillas con parasitoides, en la primera se contaron ocho parasitoides y en la segunda siete. Se tomaron fotografías y se enviaron al especialista A. V. Gumovsky en el Schmalhausen Institute of Zoology, Ucrania, para la determinación de la especie, quien indicó que se trataba de E. erythrinae (Figura 3). Se trata de una especie que Gumovsky y Ramadan (2011) describieron recientemente, indicando que es un parasitoide muy interesante ya que la larva del brúquido es parasitada en el primer estadio; para esto el brúquido coloca el huevo sobre la testa de la semilla, en ese momento comienza a realizarse la reproducción celular para formarse la larva. Una vez que se encuentra desarrollada la larva en su primer estadio dentro del corión, es cuando el parasitoide oviposita en el interior de la larva, usando el ovipositor para atravesar el corión del huevo del brúquido; posteriormente la larva del brúquido perfora la testa de la semilla y una vez adentro continúa desarrollándose, pero nunca llega a pupar, ya que antes de este proceso muere por efecto del parasitismo.

\section{DISCUSIÓN}

La cantidad de huevos ovipositados en una semilla es variable; en algunas semillas se encontraron hasta 22 hue-

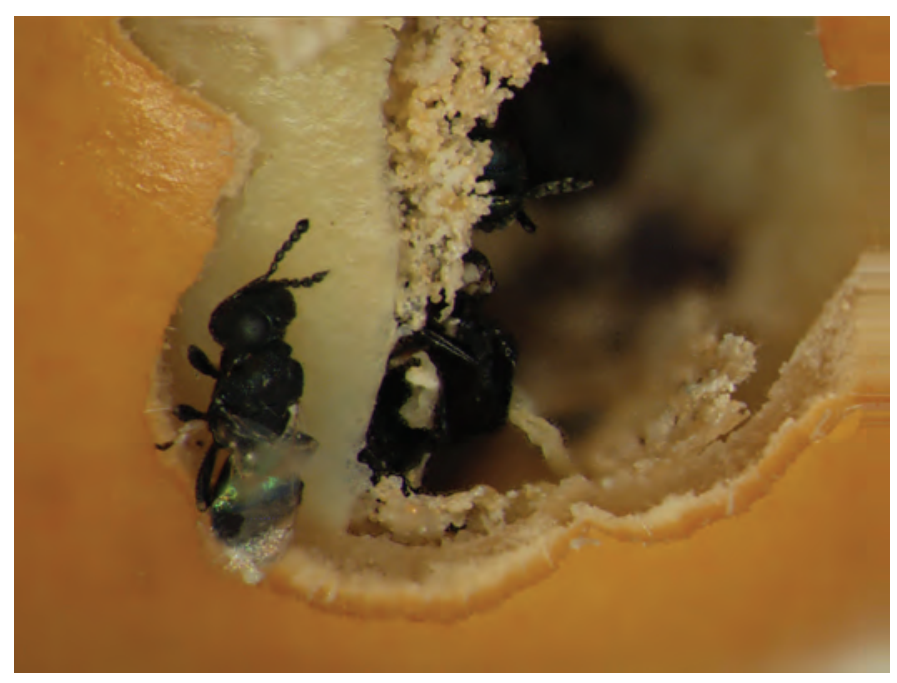

Figura 2. Adulto del parasitoide Entedeon erythrinae y algunas exuvias de pupas.

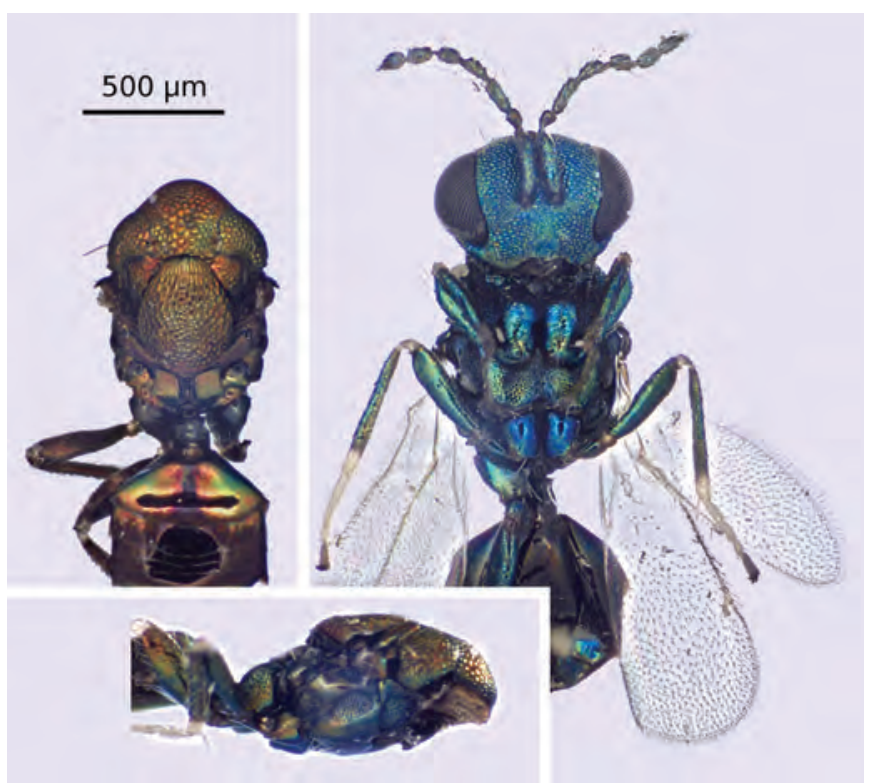

Figura 3. Adulto de Entedeon erythrinae; a) vista dorsal, b) vista lateral, c) vista ventral.

vos, sin embargo, Medeiros et al. (2008) menciona haber observado hasta 38 huevos en una semilla. En relación a la cantidad de perforaciones en la semilla y la cantidad de individuos que llegan a su desarrollo completo, se observó que presentaron hasta 12 perforaciones de individuos que llegaron a su etapa adulta. En contraste, Medeiros et al. (2008) mencionan que semillas de Erythrina sp. presentaron en promedio $4.3 \pm 2.6$ perforaciones por semilla; sin embargo, remarcan que en algunas semillas la cantidad llegaba hasta 19 en E. sandwicensis, especie nativa de Hawaii. La cantidad de emergencia de $S$ impressithorax se encuentra más alta en México que en Hawaii, quizás las condiciones del sitio han sido más favorables para el insecto.

Además de la alta cantidad de individuos que lograron emerger de la semilla, éstos tienen la facultad de reducir su tamaño en proporción a la cantidad de alimento disponible; este fenómeno también fue observado por Romero (2002) con Ctenocolum janzeni Kingsolver \& Whitehead (1991) en semillas de Lonchocarpus rugosus Benth (Leguminosae, Papilionoideae: Millettieae), detectándose casi un $100 \%$ en la reducción del cuerpo, es decir que la longitud (pronoto-élitros) promedio fue de $4.1 \mathrm{~mm}$ y en casos de escasez de alimento la talla se redujo hasta 2.1 mm de longitud. Por otro lado, Center y Johnson (1974) mencionan que una de las estrategias de los brúquidos para sobrevivir cuando existe escasez de alimento es reducir su tamaño, ya que la planta hospedera presenta reducción de las semillas; por lo tanto, la larva se desarrollará siempre que encuentre un espacio en la semilla, aún cuando el área de la misma sea menor. 
En cuanto al comportamiento de la hembra, Gumovsky y Ramadan (2011) mencionan que la hembra realiza movimientos circulares antes de ovipositar, y que el huevo es depositado directamente sobre el tegumento de la semilla, se observó este mismo comportamiento, sin embargo, la hembra pasa sus mandíbulas sobre el sitio donde será ovipositado el huevo.

Otra de las estrategias que han desarrollado estos brúquidos ha sido la alta tolerancia a los alcaloides que presentan las eritrinas (Center y Johnson 1974), lo que sugiere que la polifagia es amplia y no selectiva en cuanto a nivel de especies hospederas. Con lo anterior la lista antes publicada por Romero y Johnson (2002) de las hospederas de $S$. impressithorax se encuentra en aumento a medida que el insecto se desplaza.

Sobre la distribución actual de S. impressithorax en México, hasta el año 2012 se habían registrado los siguientes estados como parte de la expansión geográfica del insecto: Distrito Federal, Estado de México, Morelos y Veracruz; sin embargo, entre 2012 y 2013 se han registrado otras localidades en Hidalgo, Guanajuato, Jalisco, Puebla y Nayarit. Como se puede apreciar en la Figura 4, la dispersión de este brúquido se ha extendido a una velocidad muy alta; de acuerdo a Romero et al. (2009), la invasión inicial pudo haber ocurrido en el Distrito Federal y de ahí se ha propagado hacia los estados vecinos. La capacidad polífaga de este brúquido y la riqueza de especies de Erythrina en México, podrían ocasionar que se disperse por todo el país y en algunos años más se encuentre en la totalidad del continente.
En cuanto a la presencia de un parasitoide exótico en México, puede atribuirse a que al momento de ser introducido el brúquido a América, éste venía con su parasitoide natural. No obstante, el coleóptero ha tenido una mayor fecundidad y el parasitoide podría encontrarse en un proceso de adaptación, lo cual explicaría que todos los especímenes obtenidos del parasitoide hayan sido machos, y que además no hayan podido emerger por sí mismos; es posible que la emergencia de parasitoides todavía es muy reducida debido a la constitución de las semillas y a la proporción de sexos.

Agradecimientos. Al Dr. Marcos Soto Hernández del Postgrado de Botánica del Colegio de Postgraduados, por donar las semillas de Erythrina sp. para las pruebas de polifagia. Al Dr. J. Concepción Rodríguez Maciel del área de Entomología, Postgrado de Fitosanidad del Colegio de Postgraduados, por el préstamo de las cámaras de cría para hacer posible la realización de los experimentos. A la M. C. Iliana Pacheco Rueda, estudiante del programa de Entomología y Acarología, por su apoyo en la valoración de los datos. Al Ingeniero Jesús Tornero Macías por la elaboración del mapa de distribución de S. impressithorax.

\section{LITERATURA CITADA}

Bridwell, J. C. 1938. Specularius erythrinae, a new bruchid affecting seeds of Erythrina (Coleoptera). Journal of the Washington Academy of Sciences, 28: 69-76.

Center, T. D. \& Johnson, C. D. 1974. Coevolution of some seed beetles (Coleoptera: Bruchidae) and their hosts. Ecology, 55: 10961103.

Decelle, J. 1951. Contribution a l'étude des Bruchidae du Congo Bel-

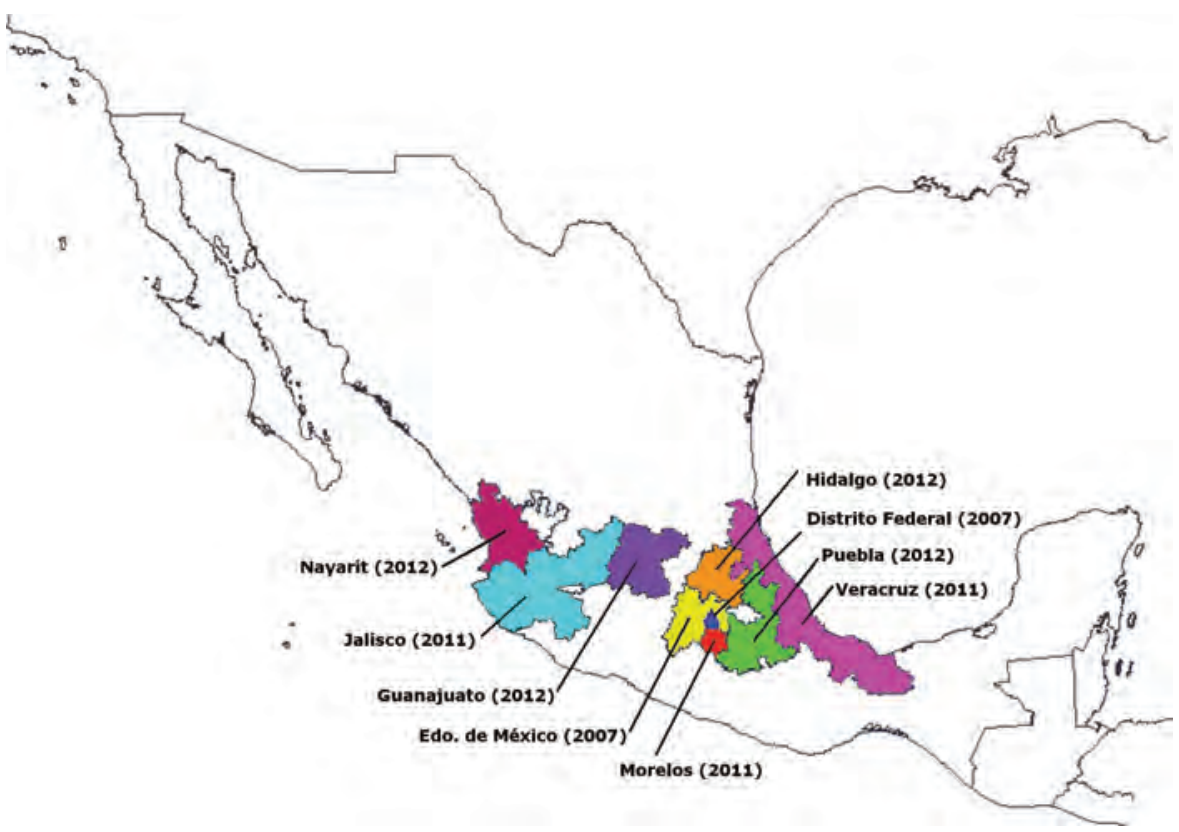

Figura 4. Mapa de distribución de Specularius impressithorax. Para cada estado se indica en paréntesis el año de registro del brúquido. 
ge (Coleoptera: Phytophaga). Revue de zoologie et de botanique africaines, 45: 172-192.

García-Mateos, M. R., Garín-Aguilar, M. E., Soto-Hernández, M. \& Martínez Vázquez, M. 2000. Effect of Erytrina americana on rats aggresive behavior. Pharmaceutical and Pharmacological Letters, 10: 34-37.

Gillett, J. B. 1962. The fruit and seeds of Erythrina brucei and the identity of E. abyssinica. Kew Bulletin, 15: 425-429.

Gulmahamad, H. 2006. First North American and California record of Specularius impressithorax (Pic) 1913, (Col.: Bruchidae) and toxicity implications of Erythrina in high profile areas. The PanPacific Entomologist, 82: 68-73.

Gumovsky, A. V. \& Ramadan, M. M. 2011. Biology, immature and adult morphology and molecular characterization of a new species of the genus Entedeon (Hymenoptera: Eulophidae) associated with the invasive pest Specularius impressithorax (Coleoptera: Chrysomelidae, Bruchinae) on Erythrina plants. Bulletin of Entomological Research, 101: 715-739.

Informe 217s. CATIE. 1989. Erythrina spp. Fase 1. Informe técnico final del proyecto. Centro Agronómico Tropical de Investigación y Enseñanza (CATIE), Costa Rica.

Kaikabo, A. A., Suleiman, M. M., Babatunde, B. S., Samuel, B. \& Eloff, N. 1988. Antibacterial activity of eleven South African Plants used in treatment of diarrhoea in folkloric medicine. Planta Medica, 64: 711-714.

Kass, D. L. 1994. Erythrina species - pantropical multipurpose tree legumes, pp. 84-96. In: R.C. Gutteridge \& H.M. Shelton (Eds.). Forage Tree Legumes in Tropical Agriculture. Wallingford, UK.

Medeiros, A. C., Vonallmen, E., Fukada, M., Samuelson, A.\& Lau, T. 2008. Impact of the newly arrived seed. Predating beetle Spe- cularius impressithorax (Coleoptera: Crysomelidae: Bruchinae) in Hawaii. Pacific Conservation Biology, 14: 1-7.

Neill, D. A. 1993. The genus Erythrina: Taxonomy, distribution and ecological differentiation, pp. 15-27. In: S. B. Westley \& M. H. Powell (Eds.). Erytrina in the new and old words. NFTA, Nitrogen Fixing tree research report special Issue.

Pic, M. 1932. Nouveautés diverses. Mélanges Exotico-Entomologiques, 59: 10-36.

Romero N., J. 2002. Bruchidae, pp. 513-534. In: J. Llorente Bousquets y Juan J. Morrone (Eds). Biodiversidad, taxonomía y biogeografía de artrópodos de México: hacia una síntesis de su conocimiento. UNAM. Vol. III.

Romero N., J. \& Johnson, C.D. 2002. Date Base BRUCOL. Programa de Entomología, Instituto de Fitosanidad, Colegio de Postgraduados, México.

Romero N., J., Kingsolver, J. M. \& Rodríguez H., C. 2009. First report of the exotic bruchid Specularius impressithorax (Pic) on seeds of Erythrina coralloides DC. in Mexico (Coleoptera: Bruchidae). Acta Zoológica Mexicana, 25: 195-198.

Ruiz, M. C., Martínez Hernández, M. de J., Romero N., J. \& Ríos Reyes, A. V. 2012. Primer reporte de Specularius impressithorax (Pic) (Coleoptera: Bruchidae) alimentándose de semillas de Erythrina americana Miller en los estados de Veracruz y Morelos, México. Acta Zoológica Méxicana (n.s.), 28: 635-639.

SAS Institute Inc. 2000. SAS/ STAT users's guide. Version 9. SAS Institute Inc, Cary, NC.

Sotelo, A., Soto, M., Lucas, B. \& Giral, F. 1993. Comparative studies of the alkaloidal composition of two Mexican Erythrina species and nutritive value of detoxified seeds. Journal of Agricultural and Food Chemistry, 41: 2340-2343. 